\title{
Editorial
}

\section{Biocompatibility and Toxicity of Nanobiomaterials 2014}

\author{
Xiaoming Li, ${ }^{1}$ Sang Cheon Lee, ${ }^{2}$ Shuming Zhang, ${ }^{3}$ and Tsukasa Akasaka ${ }^{4}$ \\ ${ }^{1}$ Key Laboratory for Biomechanics and Mechanobiology of Ministry of Education, School of Biological Science and Medical Engineering, \\ Beihang University, Beijing 100191, China \\ ${ }^{2}$ Department of Maxillofacial Biomedical Engineering, School of Dentistry, Kyung Hee University, Seoul 130-701, Republic of Korea \\ ${ }^{3}$ Department of Materials Science and Engineering, Johns Hopkins University, Baltimore, MD 21218, USA \\ ${ }^{4}$ Department of Biomedical Materials and Engineering, Hokkaido University, Sapporo 060-8586, Japan
}

Correspondence should be addressed to Xiaoming Li; x.m.li@hotmail.com

Received 21 April 2015; Accepted 21 April 2015

Copyright ( 2015 Xiaoming Li et al. This is an open access article distributed under the Creative Commons Attribution License, which permits unrestricted use, distribution, and reproduction in any medium, provided the original work is properly cited.

It is well known that nanomaterials have developed rapidly over the past few decades. Based on their unique physicochemical properties and special mechanical properties, nanomaterials have provided application possibility in many different fields. Currently, as nanobiomaterials, they are widely used in various biomedical applications, such as drug delivery systems, tissue engineering, dental/bone implant, and biosensors. For example, nanobiomaterials have been used in tissue engineering because of their satisfactory bioactivity, high mechanical properties, and large surface area to adsorb specific proteins. Many kinds of nanobiomaterials are used to prepare composite scaffolds to get better biocompatibility and higher ability in repairing specific tissues. Several antibacterial metallic nanobiomaterials are used to coat implant surfaces to improve the speed of healing fractures. In addition, lots of nanobiomaterials have the potential to break the limitations of the traditional delivery systems. They can load larger amount of drugs and provide stable drug release for long time at the targeted sites, such as tumors. Moreover, they can combine with polymers to furnish simultaneous drug delivery systems with the controllable release rate. Besides these applications, more and more nanobiomaterials show great potential to be applied as highly sensitive biosensors because they have higher ability in loading firmly or interacting completely with recognition aptamers.

Although, due to their special properties, nanobiomaterials have been applied into many aspects of the biomedical field, their biocompatibility and toxicity are very important issues, which is really the concern of more and more people. For example, the nanobiomaterials that are used to deliver drugs to targeted cells can normally traverse the cell membranes purposefully and have ineluctable interactions with intracellular substances. So, the safety of the used nanobiomaterials is fatal indeed, which directly determines the success rate of the drug delivery. Moreover, many implants containing nanomaterials undergo biodegradation in vivo and unavoidably release nanoparticles, whose effects on the cells and tissue in the physiological environment should be obviously investigated. Only when the related research findings have confirmed that the nanomaterials are safe enough for the interacted cells and surrounding tissues, can adapt to organism reciprocally, and cannot be excluded by the immune system do these nanobiomaterials have the qualification to be really used for the related biomedical purposes.

Currently, a series of in vitro and in vivo research have been launched on the biocompatibility and toxicity of nanobiomaterials. Usually, the in vitro investigations are firstly conducted to show if the materials affect the normal morphology of the cultured cells or the mixed cells with microscopies and their bioactive functions, including proliferation, differentiation, mineralization, and so forth, by studying both the genes and proteins of the cells with various biochemical analyses. After the studies in vitro, the nanobiomaterials are normally implanted into animal body, such as dorsal muscle pouch, to see if they have significant effect on the normal functions of the surrounding 
tissues and the main organs by histological, histopathological, and immunohistochemical studies. Though there have been some methods to test the biocompatibility and toxicity of nanobiomaterials, they are not enough. More appropriate methods both in vitro and in vivo to evaluate and comprehend the biocompatibility and cytotoxicity of nanobiomaterials are necessary. Most importantly, the current measurement accuracy of biocompatibility and toxicity needs further improvements. Furthermore, it is urgent to find new methods to reduce the cytotoxicity and improve the biocompatibility of nanobiomaterials.

In this special issue, several articles are devoted to show the effects of some specific nanobiomaterials on their biocompatibility and toxicity and to find out their mechanisms. For example, the investigations into the biocompatibility of nanohydroxyapatite coated magnetic nanoparticles under magnetic situation, the effects of surface properties of nanostructured bone repair materials on their performances, and the transport and deposition of nanoparticles in respiratory system by inhalation also have been studied. Deep investigations into the applications of nanobiomaterials have been carried out, such as their recent applications in prosthodontics. In addition, accurate data and updated reviews about the preparation, synthesis, properties, and evaluations of nanobiomaterials have been presented. For instance, the preparation and in vitro evaluations of a nanoscaled injectable bone repair material and the synthesis of luminescent Ag nanoclusters with antibacterial activity have been studied. What is more, some researches focused on the clinical effects of nanobiomaterials. For example, the clinical use of the nanohydroxyapatite/polyamide mesh cage in anterior cervical corpectomy and fusion surgery and the clinical effects of novel nanoscaled core decompression rods combined with umbilical cord mesenchymal stem cells on the treatment of early osteonecrosis of the femoral head have been investigated. This is a successful issue to cover many aspects of evaluating the biocompatibility and toxicity of nanobiomaterials in vitro and in vivo. However, further researches on this subject are still needed, such as investigations into the effects of size, shape, and surface of nanobiomaterials on their biocompatibility and toxicity and more deep analytical approaches to animal experiments and much more convincing mechanisms of the corresponding researches.

Xiaoming $L i$

Sang Cheon Lee

Shuming Zhang

Tsukasa Akasaka 

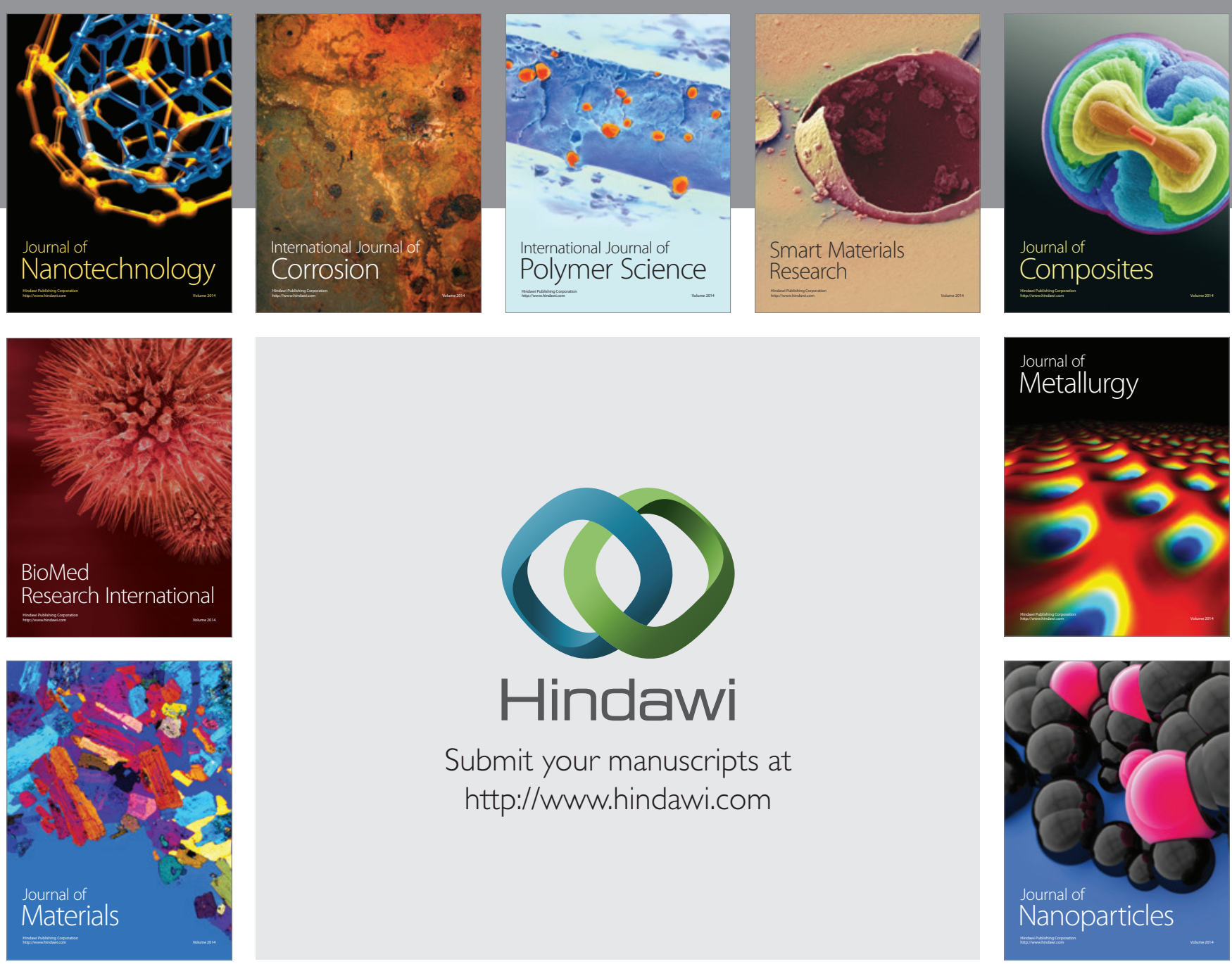

Submit your manuscripts at http://www.hindawi.com
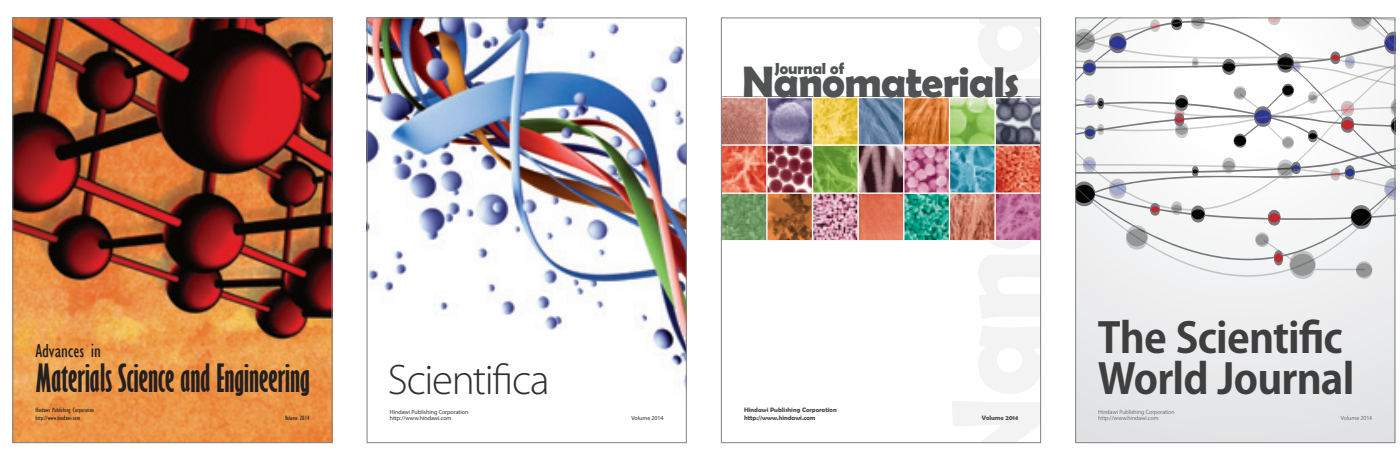

\section{The Scientific World Journal}
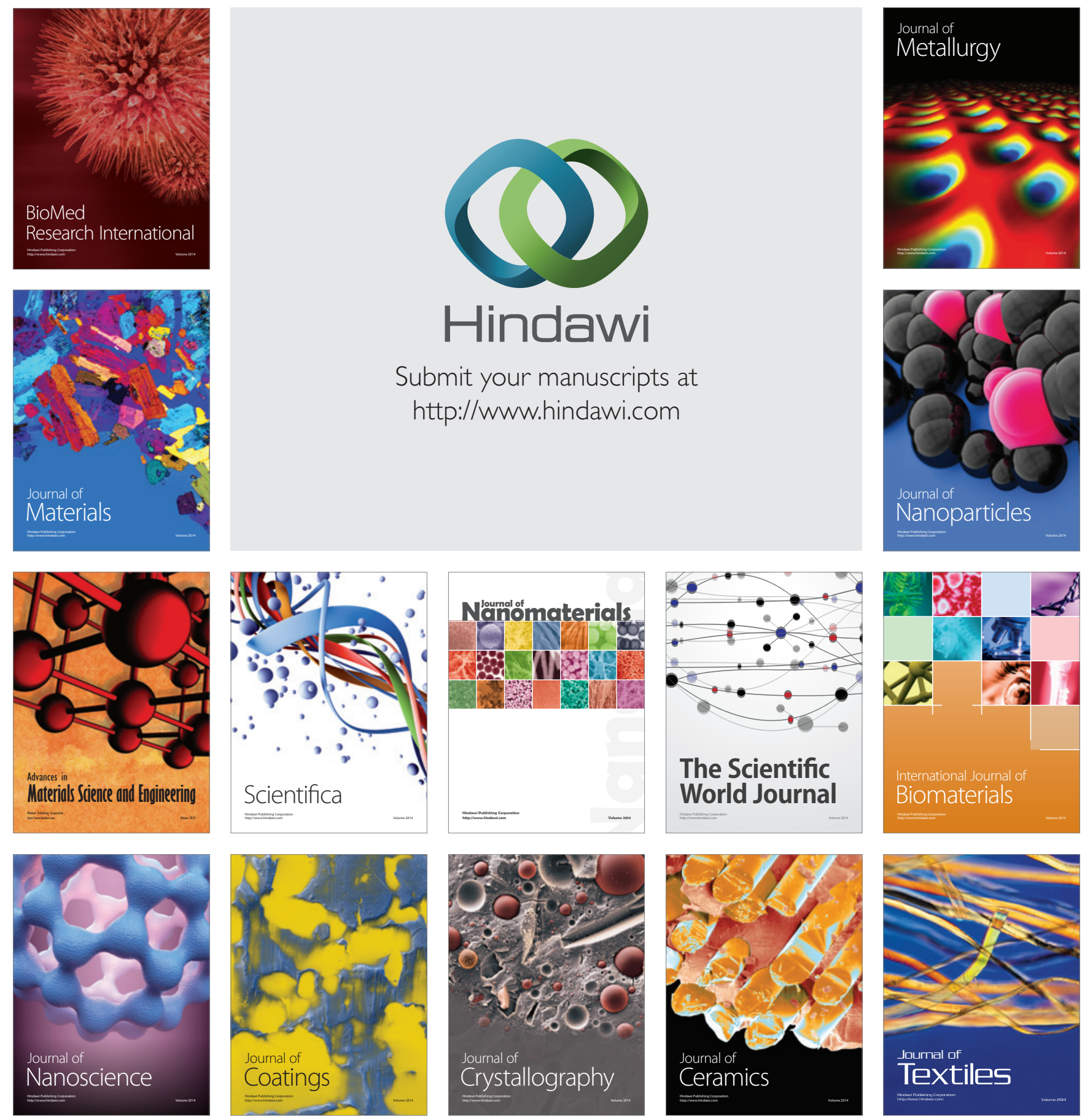